\title{
Mechanisms of CYP3A Induction by Glucocorticoids in Human Fetal Liver Cells
}

\author{
Tamihide Matsunaga ${ }^{1,2}$, Masataka Maruyama ${ }^{1,3}$, Tsutomu Matsubara ${ }^{4,5}$, \\ Kiyoshi Nagata ${ }^{6}$, Yasushi Yamazoe ${ }^{4}$ and Shigeru Ohmorl ${ }^{1, *}$ \\ ${ }^{1}$ Department of Pharmacy, Shinshu University Hospital, Matsumoto, Japan \\ ${ }^{2}$ Department of Clinical Pharmacy, Graduate School of Pharmaceutical Sciences, Nagoya City University, \\ Nagoya, Japan \\ ${ }^{3}$ Department of Pharmacy, Fujimi Kogen Hospital, Nagano, Japan \\ ${ }^{4}$ Division of Drug Metabolism and Molecular Toxicology, Graduate School of Pharmaceutical Sciences, \\ Tohoku University, Sendai, Japan \\ ${ }^{5}$ Department of Anatomy, Graduate School of Medicine, Osaka City University, Osaka, Japan \\ ${ }^{6}$ Division of Environmental Health, Tohoku Pharmaceutical University, Sendai, Japan
}

Full text of this paper is available at http://www.jstage.jst.go.jp/browse/dmpk

\begin{abstract}
Summary: Human fetal liver (HFL) cells express major drug metabolic enzymes CYP3A4, CYP3A5 and CYP3A7. In the fetal hepatocytes, betamethasone and dexamethasone (DEX) markedly enhanced the expression levels of CYP3A4 and CYP3A7 mRNAs and slightly increased the expression level of CYP3A5 mRNA. Interestingly, a high correlation between the CYP3A induction ability and the intensity of antiinflammatory effect was observed. Human glucocorticoid receptor (GR)-small interfering RNA clearly attenuated the expression level of GR mRNA, and diminished the DEX-stimulated CYP3A4, CYP3A5 and CYP3A7 expression in HFL cells. These findings indicate that GR mediates the induction of CYP3A4 and CYP3A7 expression in human fetal hepatocytes as well as the CYP3A5.
\end{abstract}

Keywords: CYP3A; induction; glucocorticoid; human fetal liver cells; glucocorticoid receptor; small interfering RNA; specific small interfering RNA

\section{Introduction}

Cytochrome P450 (CYP) comprises a gene superfamily of hemoproteins that catalyze the oxidation of lipophilic substrates to more water-soluble products. One of them, the human CYP3A subfamily, contains mainly four isoforms, CYP3A4, CYP3A5, CYP3A7, and CYP3A43. In particular, CYP3A4, CYP3A5 and CYP3A7, highly expressed in liver, associate with the metabolism of many compounds. The expression levels of CYP3A isoforms are enhanced by treatment with various agents, such as rifampicin (RIF), phenobarbital, clotrimazole, and dexamethasone (DEX). ${ }^{1-3)}$ We previously clarified that CYP3A4 and CYP3A7 mRNA expression levels were markedly up-regulated by DEX, but not by RIF, in human fetal liver (HFL) cells. ${ }^{4)}$ These data suggested that the mechanisms of CYP3A induction in HFL differed from those in adult liver.
The glucocorticoid-induced CYP3A5 expression is mediated by glucocorticoid receptor (GR) signaling. ${ }^{5,6}$ ) As previously described, transcriptional activation of the CYP $3 A 4$ gene by glucocorticoids is also known to occur through two distinct mechanisms involving GR: first by controlling the expression of pregnane $\mathrm{X}$ receptor (PXR) under physiological conditions through the classical GR pathway, and second by activating PXR under bolus or stress conditions. ${ }^{7,8)}$ The role of GR in CYP3A4 regulation is, however, unclear, and an accurate assessment of whether GR plays a direct and/or indirect role remains obscure. ${ }^{9)} \mathrm{We}$ have reported that concomitant treatment with RU486, a GR antagonist, suppressed DEX-mediated induction of CYP3A4, CYP3A5, and CYP3A7 expression completely in HFL cells. ${ }^{4)}$ These data suggested that GR was required in the CYP3A4 induction.

Introduction of specific small interfering RNA (siRNA) in cells has been shown to specifically knock down the target

Received February 4, 2012; Accepted May 7, 2012

J-STAGE Advance Published Date: May 22, 2012, doi:10.2133/dmpk.DMPK-12-NT-018

*To whom correspondence should be addressed: Shigeru OHмORI, Ph.D., Department of Pharmacy, Shinshu University Hospital, 3-1-1 Asahi, Matsumoto 390-8621, Japan. Tel. +81-263-37-2826, Fax.+81-263-37-2826, E-mail address: somori@shinshu-u.ac.jp

This study was partly supported by Grants-in-Aid for Scientific Research (encouragement study) from the Ministry of Education, Culture, Sports, Science and Technology, Japan (Nos. 15390047, 16590109). 
gene expression. ${ }^{10)}$ Recently, Matsubara et al. ${ }^{11)}$ reported that adenovirus vector expressing human PXR-siRNA (AdhPXR-siRNA) was a potent tool to discern the role of PXR in the chemical-mediated activation of the CYP3A4 gene. The system could be useful for assessment of a variety of nuclear receptor functions in vivo and in vitro.

In the present study, to further characterize whether GR is involved in DEX-mediated activation of CYP3A4 and CYP $3 A 7$ genes as well as the CYP $3 A 5$ gene, we investigated the effect of glucocorticoids on the CYP3A expression and the role of GR in the induction of the CYP3A gene expression in HFL cells using the AdhGR-siRNA system.

\section{Materials and Methods}

Materials: SuperScript II first-strand synthesis system for reverse transcription-polymerase chain reaction (RTPCR) and TRIzol reagent were purchased from Invitrogen (Carlsbad, CA, USA); DEX, hydrocortisone, prednisolone and betamethasone were obtained from Wako Pure Chemicals (Osaka, Japan); fludrocortisone and methylprednisolone were obtained from MP Biomedicals (Costa Mesa, CA, USA); cortisone and Williams' medium E were obtained from Sigma Chemical Co. (St. Louis, MO, USA); TaKaRa EX Taq was obtained from Takara-Bio (Otsu, Japan); and PCR primers were purchased from Sigma Genosys (Hokkaido, Japan). All other reagents used were of the highest quality available.

Human fetal liver cells: HFL cells were obtained from Applied Cell Biology Research Institute (Kirkland, WA, USA). The cell culture was initiated from a pool of six normal human liver tissues (average gestation 13 weeks) by elutriation following dispase digestion of tissue. The cell culture was cryopreserved at $-150^{\circ} \mathrm{C}$ until use after proliferation.

Cell culture and drug treatment: HFL cells were incubated on dishes coated with type I collagen. Williams' medium E (Sigma Chemical Co.) containing 10\% (v/v) fetal bovine serum, antibiotics $(50 \mu \mathrm{g} / \mathrm{mL}$ penicillin, $50 \mu \mathrm{g} / \mathrm{mL}$ streptomycin, and $100 \mu \mathrm{g} / \mathrm{mL}$ neomycin), and $2 \mathrm{mM} \mathrm{L}$ glutamine was used for culture of $\mathrm{HFL}$ cells under the condition of $5 \% \mathrm{CO}_{2}$ at $37^{\circ} \mathrm{C}$. The medium was exchanged every $24 \mathrm{~h}$.

Induction of CYP3A mRNA by representative glucocorticoids: HFL cells were cultured for 7 days after seeding of 5,000 cells/well onto 6-well culture plates, and then treated with $10 \mathrm{nM}$ cortisone, hydrocortisone, prednisolone, methylprednisolone, fludrocortisone, betamethasone or DEX for $72 \mathrm{~h}$. The compounds were dissolved in dimethyl sulfoxide (DMSO), which was added to the culture medium at a final concentration of $0.1 \%$. The medium was replaced daily with fresh medium containing either test compounds dissolved in vehicle or vehicle alone. After treatment, total RNA was prepared from the cells.

Construction of siRNA-expressing adenovirus: Human H1 RNA gene promoter was used for the expression of siRNA. AdhGR-siRNA was constructed with AdEasy ${ }^{\mathrm{TM}}$ System (MP Biomedicals, Irvine, CA, USA) according to the manufacturer's protocol. The hGR-specific siRNA, designed by Takara-Bio, was amplified by PCR with primers $5^{\prime}$ CGCGTCGACATGACCCTACTGCAGTACTTTCAAGAGAAG-3' and 5'-CGCAAGCTTAAAAAAATGACCCTACTGCAGTACTTCTCTTGAAAG-3'. PCR product was digested with Sal I and Hind III, and ligated into the same restriction sites of pShattle-H1. AdCont (AxCALacZ), which expresses $\beta$-galactosidase, was provided by Dr. Izumi Saito (Tokyo University). The titer of adenovirus, $50 \%$ titer culture infections dose $\left(\mathrm{TCID}_{50}\right)$, was determined in HEK293 cells. The value of $\mathrm{TCID}_{50}$ was reported to be almost equivalent to that of plaque-forming units. ${ }^{12)}$ Multiplicity of infection (MOI) was calculated by dividing $\mathrm{TCID}_{50}$ by the number of cells.

Effects of GR knockdown by adenoviral hGRsiRNA expression on CYP3A induction by DEX: HFL cells were cultured for 1 day after seeding 100,000 cells/well onto 6-well culture plates, and then were infected with AdhGR-siRNA (MOI of 10 or 50). The HFL cells cultured for 3 days after infection were incubated with Williams' medium E containing vehicle (0.1\% DMSO) or $100 \mathrm{nM}$ DEX for 2 days. We used AdCont as a control for RNA knockdown experiments. Thereafter, total RNA was prepared from HFL cells and CYP3A mRNA was analyzed by semiquantitative RT-PCR.

RNA extraction and semiquantitative RT-PCR analysis: Total RNA was extracted from the treated cells with TRIzol reagent (Invitrogen, Carlsbad, CA, USA). Firststrand cDNA was generated from $2 \mu \mathrm{g}$ total RNA. Reversetranscription reaction was performed using a SuperScript II (Invitrogen) according to the manufacturer's instructions. With the cDNA obtained, PCR was carried out using a MyCycler thermal cycler (Bio-Rad, Hercules, CA, USA) and PCR Express thermal cycler (Hybaid, Middlesex, UK). The primers and amplification conditions used are summarized in Table 1. The amplified products were separated by $2 \%$ agarose gel electrophoresis and stained with ethidium bromide. The levels of these mRNAs were quantified from their band densities on the agarose gels using Printgraph AE-6914 and Scion Image Software (by Dr. W. Rasband, http://www.scioncorp.com/), and were normalized relative to that of glyceraldehyde-3-phosphate dehydrogenase (GAPDH) mRNA.

\section{Results and Discussion}

Effects of representative glucocorticoids on expression of CYP3A mRNA: Anti-inflammatory effects of glucocorticoids are caused by binding of GR homodimers to glucocorticoid response elements in the promoter region of steroid-sensitive genes, which may encode anti-inflammatory proteins. ${ }^{13)}$ If GR mediates the CYP3A induction, the glucocorticoid-mediated change in CYP3A expression could be highly correlated with the anti-inflam- 
Table 1. PCR primers and conditions

\begin{tabular}{lcll}
\hline \hline Primer & $\begin{array}{c}\text { Length/ } \\
\text { annealing temperature }\end{array}$ & \multicolumn{1}{c}{ Sense primer $5^{\prime} \rightarrow 3^{\prime}$} & Antisense primer $5^{\prime} \rightarrow 3^{\prime}$ \\
\hline CYP3A 4 & $626 \mathrm{bp} / 60^{\circ} \mathrm{C}$ & CTGTGTGTTTCCAAGAGAAGTTAC & ACCTCATGCCAATGCAGTTT \\
CYP3A5 & $239 \mathrm{bp} / 62^{\circ} \mathrm{C}$ & TGACCCAAAGTACTGGACAG & TGAAGAAGTCCTTGCGTGTC \\
CYP3A7 & $475 \mathrm{bp} / 54^{\circ} \mathrm{C}$ & CTATGATACTGTGCTACAGT & TCAGGCTCCACTTACGGTCT \\
GR & $557 \mathrm{bp} / 54^{\circ} \mathrm{C}$ & ACACAGGCTTCAGGTATCTT & ACTGCTTCTGTTGCCAAG \\
PXR & $442 \mathrm{bp} / 68^{\circ} \mathrm{C}$ & CAAGCGGAAGAAAAGTGAACG & CTGGTCCTCGATGGGCAAGTC \\
GAPDH & $307 \mathrm{bp} / 54^{\circ} \mathrm{C}$ & CATCACCATCTTCCAGGAGC & CATGAGTCCTTCCACGATACC \\
\hline
\end{tabular}

Table 2. Relative anti-inflammatory potencies of representative glucocorticoids and their ability to induce CYP3As

\begin{tabular}{lcccc}
\hline \hline \multirow{2}{*}{ Compound } & \multirow{2}{*}{$\begin{array}{c}\text { Anti-inflammatory } \\
\text { potency }^{a}\end{array}$} & \multicolumn{3}{c}{ Induction ability (CYP3As/GAPDH) } \\
\cline { 3 - 5 } & - & $1.00 \pm 0.02$ & $1.00 \pm 0.02$ & $1.00 \pm 0.02$ \\
& CYP3A4 & CYP3A5 & CYP3A7 \\
\hline Control (DMSO) & 0.8 & $0.67 \pm 0.05$ & $0.63 \pm 0.05$ & $0.91 \pm 0.26$ \\
Cortisone & $\mathbf{1}$ & $\mathbf{0 . 7 1} \pm \mathbf{0 . 0 5}$ & $\mathbf{0 . 6 2} \pm \mathbf{0 . 0 2}$ & $\mathbf{1 . 1 0} \pm \mathbf{0 . 1 8}$ \\
Hydrocortisone & 4 & $1.38 \pm 0.23$ & $0.93 \pm 0.03$ & $2.41 \pm 0.65$ \\
Prednisolone & 5 & $1.53 \pm 0.12$ & $0.49 \pm 0.05$ & $1.73 \pm 0.54$ \\
Methylprednisolone & 10 & $1.82 \pm 0.30$ & $0.64 \pm 0.08$ & $2.43 \pm 0.57$ \\
Fludrocortisone & 25 & $2.90 \pm 0.51$ & $1.75 \pm 0.16$ & $4.33 \pm 0.80$ \\
Betamethasone & 25 & $3.75 \pm 1.27$ & $1.87 \pm 0.09$ & $3.79 \pm 1.60$ \\
Dexamethasone & & & &
\end{tabular}

${ }^{a}$ Relative to hydrocortisone, which is assigned a value of $1 .{ }^{13}$

${ }^{b} \mathrm{HFL}$ cells were cultured for 7 days after seeding 5,000 cells/well onto 6-well culture plates, and then treated with $10 \mathrm{nM}$ glucocorticoids for $72 \mathrm{~h}$. After treatment, total RNA was prepared from the cells. The mRNA levels were calculated using semiquantitative RT-PCR analysis as described in Materials and Methods. The values are expressed as the mean \pm standard deviation of three experiments.

matory effect of the glucocorticoids. Thus we investigated whether representative glucocorticoids used in clinical medicine induce CYP3A4, CYP3A5 and CYP3A7 expression in HFL cells (Table 2). The levels of CYP3A4 and CYP3A7 mRNAs were markedly enhanced by treatment with $10 \mathrm{nM}$ of betamethasone or DEX, which are classified as the most potent glucocorticoids. The expression level of CYP3A5 mRNA was also enhanced, but the induction of CYP3A5 expression was slight, compared to that of the CYP3A4 and CYP3A7. Generally, a good correlation has been found between the affinity for the cytosolic GR and antiinflammatory potencies of glucocorticoids. ${ }^{14)}$ Interestingly, a high correlation between the inducibility and the antiinflammatory potencies of glucocorticoids ${ }^{15}$ ) was observed, especially for CYP3A4 $\left(r^{2}=0.92\right)$ and CYP3A7 $\left(r^{2}=0.91\right)$ (Fig. 1). These results may indicate that GR mediates the induction of CYP3As in HFL cells. Glucocorticoids might induce the expression of CYP3As by complexing with GR, undergoing nuclear translocation, interacting with DNA cis sequences and modulating gene transcription.

Effects of adenoviral hGR-siRNA expression on CYP3A induction by DEX: Expression levels of CYP3A4, CYP3A5 and CYP3A7 mRNAs were increased 6.9-, 1.6- and 5.3-fold, respectively, of the control by treatment with $100 \mathrm{nM}$ of DEX (Figs. 2A and 2C).

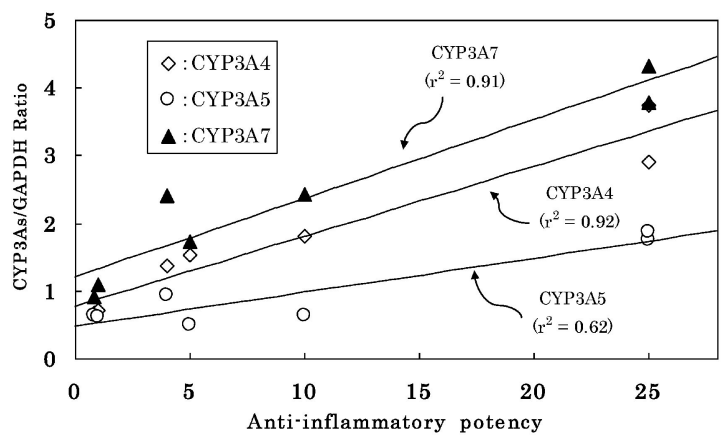

Fig. 1. Correlation between the anti-inflammatory potencies of glucocorticoids and the CYP3A-induction ability

The molecular mechanism of induction is best understood for CYP3A4. The first $1 \mathrm{~kb}$ of the $5^{\prime}$-flanking regions of CYP3A4 and CYP3A7 share $91 \%$ sequence similarity. ${ }^{16}$ ) However, the $5^{\prime}$-flanking region of CYP3A5 ( -1 to $-1,434$ bp) shares 60 and $59 \%$ sequence similarity to that of CYP3A4 and CYP3A7, respectively. ${ }^{17)}$ The low homology might be one of the factors of weak induction of CYP3A5.

To discern the involvement of GR in the induction process of CYP3As by DEX, AdhGR-siRNA was introduced to specifically knock down the target gene expression in the cells (Fig. 2). GR mRNA was detected in HFL cells and the expression was suppressed to less than $50 \%$ of that in the control by the expression of hGR-siRNA (Figs. 2A and 2B). Introduction of AdhGR-siRNA almost completely inhibited the DEX-mediated induction of CYP3A4 and CYP3A5 mRNAs (Fig. 2C). On the other hand, the induction of CYP3A7 mRNA was suppressed to about $60 \%$ of AdCont used as a control for RNA knockdown experiments. We do not know the reason why the induction of CYP3A7 mRNA was not completely suppressed by hGR-siRNA (Fig. 2C). This phenomenon might be caused by the different structure of the $5^{\prime}$-flanking regions of CYP3A4 and CYP3A7, including GR responsive elements.

Chemical-induced expression of the CYP3A4 gene can be mediated by PXR heterodimerized with retinoid $\mathrm{X}$ receptor through binding to the CYP $3 A 45^{\prime}$-flanking region. ${ }^{18-20)}$ In the present study, both 10 and $100 \mathrm{nM}$ of DEX showed clear induction of CYP3A4 and CYP3A7 expression in HFL cells (Table 2 and Fig. 2C), concentrations sufficient to activate GR but not PXR. ${ }^{7}$ We have reported that PXR mRNA is 
A

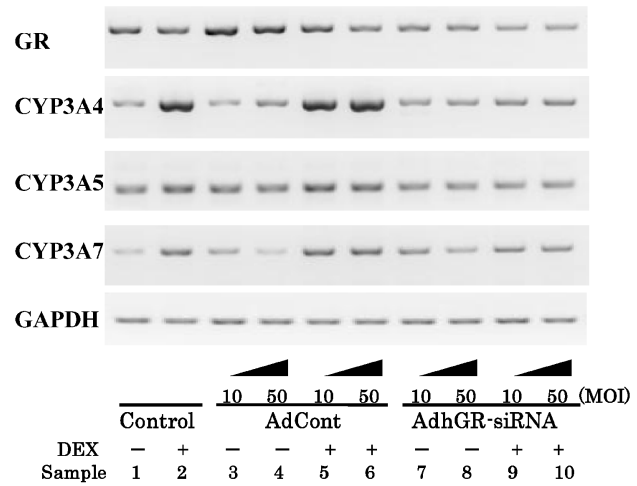

$\mathrm{B}$

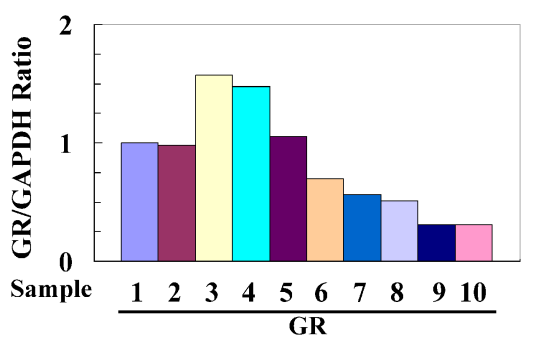

$\mathrm{C}$

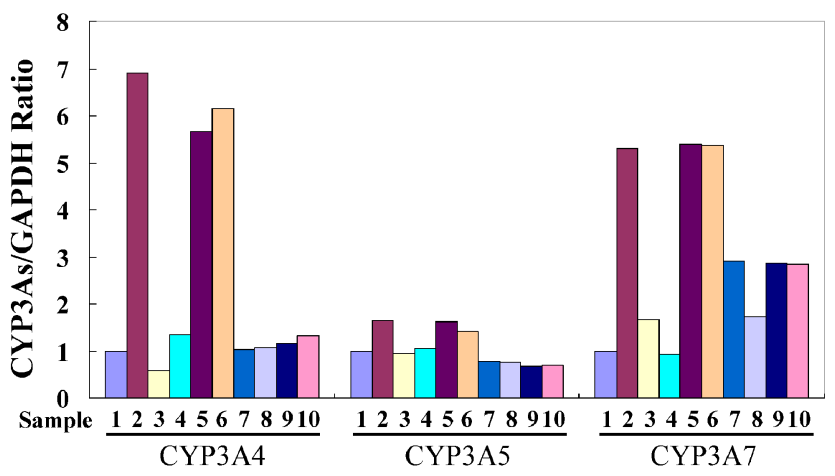

Fig. 2. (Color online) Effects of adenoviral GR-siRNA expression on GR, CYP3A4, CYP3A5 and CYP3A7 mRNA levels

HFL cells were cultured for 1 day after seeding 100,000 cells/well onto 6-well culture plates, and then were infected with AdhGRsiRNA (MOI of 10 or 50 ). We used AdCont as a control for RNA knockdown experiments. The HFL cells cultured for 3 days after infection were incubated with Williams' medium $E$ containing vehicle ( $0.1 \%$ DMSO) or $100 \mathrm{nM}$ DEX for 2 days. Then, total RNA was prepared from HFL cells and GR, CYP3A4, CYP3A5 and CYP3A7 mRNAs were analyzed by semiquantitative RT-PCR as described in Materials and Methods. A: An image of ethidium bromide-stained agarose gel is shown. Data presented are the ratio of $B$ : GR, and C: CYP3A4, CYP3A5, and CYP3A7 mRNA to GAPDH normalized at 1.0 for DMSO treatment alone in the absence of adenovirus (Sample 1). The columns present the mean of two individual experiments.

not detected by RT-PCR in HFL cells. ${ }^{21)}$ Furthermore, insufficient RIF-mediated CYP3A4 induction was observed in HFL cells with only PXR overexpression, maybe because of lower expression of some crucial transcription factors such as hepatocyte nuclear factor $4 \alpha$ (HNF4 $\alpha)$ and peroxisome proliferator-activated receptor $\gamma$ coactivator $1 \alpha$ $(\mathrm{PGC} 1 \alpha)$ in the HFL cells. ${ }^{22}$ More recently, Pang et al. reported that glucocorticoids, including dexamethasone, cortisol, corticosterone, and cortisone, all induced the expression of CYP3A7 mRNA, whereas RIF had no effect on CYP3A7 expression in double-transgenic mice expressing human PXR and CYP3A4/7. They suggested that CYP3A7 is developmentally regulated in mouse liver primarily by glucocorticoids through the GR. ${ }^{23)}$ These results strongly support a view that DEX induces CYP3A expression through GR, but not PXR, in human fetal liver.

\section{References}

1) Meunier, V., Bourrie, M., Julian, B., Marti, E., Guillou, F., Berger, Y. and Fabre, G.: Expression and induction of CYP1A1/ 1A2, CYP2A6 and CYP3A4 in primary cultures of human hepatocytes: a 10-year follow-up. Xenobiotica, 30: 589-607 (2000).

2) Luo, G., Cunningham, M., Kim, S., Burn, T., Lin, J., Sinz, M., Hamilton, G., Rizzo, C., Jolley, S., Gilbert, D., Downey, A., Mudra, D., Graham, R., Carroll, K., Xie, J., Madan, A., Parkinson, A., Christ, D., Selling, B., LeCluyse, E. and Gan, L. S.: CYP3A4 induction by drugs: correlation between a pregnane $\mathrm{X}$ receptor reporter gene assay and CYP3A4 expression in human hepatocytes. Drug Metab. Dispos., 30: 795-804 (2002).

3) Madan, A., Graham, R. A., Carroll, K. M., Mudra, D. R., Burton, L. A., Krueger, L. A., Downey, A. D., Czerwinski, M., Forster, J., Ribadeneira, M. D., Gan, L. S., LeCluyse, E. L., Zech, K., Robertson, P., Jr., Koch, P., Antonian, L., Wagner, G., Yu, L. and Parkinson, A.: Effects of prototypical microsomal enzyme inducers on cytochrome $\mathrm{P} 450$ expression in cultured human hepatocytes. Drug Metab. Dispos., 31: 421-431 (2003).

4) Matsunaga, T., Maruyama, M., Harada, E., Katsuyama, Y., Sugihara, N., Ise, H., Negishi, N., Ikeda, U. and Ohmori, S.: Expression and induction of CYP3As in human fetal hepatocytes. Biochem. Biophys. Res. Commun., 318: 428-434 (2004).

5) Schuetz, J. D., Schuetz, E. G., Thottassery, J. V., Guzelian, P. S., Strom, S. and Sun, D.: Identification of a novel dexamethasone responsive enhancer in the human CYP3A5 gene and its activation in human and rat liver cells. Mol. Pharmacol., 49: 63-72 (1996).

6) Hukkanen, J., Vaisanen, T., Lassila, A., Piipari, R., Anttila, S., Pelkonen, O., Raunio, H. and Hakkola, J.: Regulation of CYP3A5 by glucocorticoids and cigarette smoke in human lung-derived cells. J. Pharmacol. Exp. Ther., 304: 745-752 (2003).

7) Pascussi, J. M., Drocourt, L., Gerbal-Chaloin, S., Fabre, J. M., Maurel, P. and Vilarem, M. J.: Dual effect of dexamethasone on CYP3A4 gene expression in human hepatocytes: sequential role of glucocorticoid receptor and pregnane X receptor. Eur. J. Biochem., 268: 6346-6358 (2001).

8) Pascussi, J. M., Gerbal-Chaloin, S., Drocourt, L., Maurel, P. and Vilarem, M. J.: The expression of CYP2B6, CYP2C9 and CYP3A4 genes: a tangle of networks of nuclear and steroid receptors. Biochim. Biophys. Acta, 1619: 243-253 (2003).

9) Gibson, G. G., Plant, N. J., Swales, K. E., Ayrton, A. and El-Sankary, W.: Receptor-dependent transcriptional activation of cytochrome P4503A genes: induction mechanisms, species differences and interindividual variation in man. Xenobiotica, 32: 165206 (2002).

10) Elbashir, S. M., Harborth, J., Lendeckel, W., Yalcin, A., Weber, K. and Tuschl, T.: Duplexes of 21-nucleotide RNAs mediate RNA interference in cultured mammalian cells. Nature, 411: 494 498 (2001).

11) Matsubara, T., Noracharttiyapot, W., Toriyabe, T., Yoshinari, K., 
Nagata, K. and Yamazoe, Y.: Assessment of human pregnane X receptor-involvement in pesticide-mediated activation of CYP3A4 gene. Drug Metab. Dispos., 35: 728-733 (2007).

12) Kanegae, Y., Makimura, M. and Saito, I.: A Simple and efficient method for purification of infectious recombinant adenovirous. Jpn. J. Med. Sci. Biol., 47: 157-166 (1994).

13) Barnes, P. J.: Corticosteroid effects on cell signaling. Eur. Respir. J., 27: 413-426 (2006).

14) Ponec, M., Kempenaar, J., Shroot, B. and Caron, J. C.: Glucocorticoids: binding affinity and lipophilicity. J. Pharm. Sci., 75: 973-975 (1986).

15) Schimmer, B. P. and Parker, K. L.: Adrenocorticotropic hormone; adrenocortical steroids and their synthetic analogs; inhibitors of the synthesis and actions of adrenocortical hormones. In Brunton, L. L., Lazo, J. S. and Parker, K. L. (eds.), Goodman and Gilman's The Pharmacological Basis of Therapeutics, 11th ed., New York, McGraw-Hill, 2006, pp. 1587-1612.

16) Hashimoto, H., Toide, K., Kitamura, R., Fujita, M., Tagawa, S., Itoh, S. and Kamataki, T.: Gene structure of CYP3A4, an adultspecific form of cytochrome $\mathrm{P} 450$ in human livers, and its transcriptional control. Eur. J. Biochem., 218: 585-595 (1993).

17) Jounaïdi, Y., Guzelian, P. S., Maurel, P. and Vilarem, M. J.: Sequence of the 5 -flanking region of CYP3A5: comparative analysis with CYP3A4 and CYP3A7. Biochem. Biophys. Res. Commun., 205: 1741-1747 (1994).

18) Bertilsson, G., Heidrich, J., Svensson, K., Asman, M., Jendeberg,
L., Sydow-Backman, M., Ohlsson, R., Postlind, H., Blomquist, P. and Berkenstam, A.: Identification of a human nuclear receptor defines a new signaling pathway for CYP3A induction. Proc. Natl. Acad. Sci. USA, 95: 12208-12213 (1998).

19) Blumberg, B., Sabbagh, W., Jr., Juguilon, H., Bolado, J., Jr., van Meter, C. M., Ong, E. S. and Evans, R. M.: SXR, a novel steroid and xenobiotic-sensing nuclear receptor. Genes Dev., 12: 3195-3205 (1998).

20) Lehmann, J. M., McKee, D. D., Watson, M. A., Willson, T. M., Moor, J. T. and Kliewer, S. A.: The human orphan nuclear receptor PXR is activated by compounds that regulate CYP3A4 gene expression and cause drug interactions. J. Clin. Invest., 102: 1016-1023 (1998).

21) Maruyama, M., Matsunaga, T., Harada, E. and Ohmori, S.: Comparison of basal gene expression and induction of CYP3As in HepG2 and human fetal liver cells. Biol. Pharm. Bull., 30: 20912097 (2007).

22) Takezawa, T., Matsunaga, T., Aikawa, K., Nakamura, K. and Ohmori, S.: Lower expression of $\mathrm{HNF} 4 \alpha$ and PGC1 $\alpha$ might impair rifampicin-mediated CYP3A4 induction under conditions where PXR overexpressed in human fetal liver cells. Drug Metab. Pharmacokinet., 27: 430-438 (2012).

23) Pang, X. Y., Cheng, J., Kim, J. H., Matsubara, T., Krausz, K. W. and Gonzalez, F. J.: Expression and regulatjion of human fetalspecific CYP3A7 in mice. Endocrinology, 153: 1453-1463 (2012). 